\title{
LA CIENCIA EN EL FINAL DE LA EDAD MEDIA
}

IGNACIO VERDÚ BERGANZA

Universidad Pontificia Comillas.

\begin{abstract}
RESUMEN: El presente artículo pretende mostrar que, en el ámbito de la ciencia, la Edad Media no fue un período oscuro y sin avances significativos, y que la modernidad no supuso una ruptura con lo hecho al final del medievo, sino algo muy distinto. Decir lo contrario es mantener un tópico que desfigura la realidad.

PALABRAS CLAVE: Aristóteles, innovación, Grosseteste, ruptura, Bradwardine, aritmética, algebra, Merton College, Trigonometría, Oresme, Física, Buridán, impetus, Ockham.
\end{abstract}

\section{The science at the end of the Middle Ages}

ABSTRACT: TThis article aims to show that, in what concerns science, the Middle Ages were far from obscure and marked by lack scientific progress. The event of modernity did not entail a break with science at the end of the Middle Ages, but something very diferent. To keep on asserting the existence of a break represents reiterating without any real foundations.

KEY WORDS: Aristotle, innovation, Grosseteste, rupture, Bradwardine, arithmetic, algebra, Merton College, trigonometry, Oresme, Physics, Buridan, ímpetus, Ockham.

Abordar en un artículo como éste un asunto tan complejo como el de cuál era el estado de la ciencia, sus logros y progresos, al final de la Edad Media, así como la influencia que éstos pudieron ejercer en lo que conocemos como la modernidad, es una tarea nada fácil, pero, sin duda, estimulante.

Entre el siglo XIII y el xIV se produjeron extraordinarios avances, cambios, progresos, rupturas, desarrollos, polémicas y descubrimientos en todos los ámbitos de la cultura. Esto se constata en la Política, la Teología, la Lógica, la Metafísica, el Arte (arquitectura, escultura, pintura, literatura, música) y, como no, en la Ciencia (Filosofía de la Naturaleza).

Hasta tal punto es esto así que no pocos estudiosos del periodo han situado en éste el final de la Edad Media y el comienzo de una nueva era; la de Petrarca o Gioto, la del comienzo de la matematización de la naturaleza y la secularización-laicización del mundo ${ }^{1}$.

En efecto, la cuestión de la continuidad o no entre lo hecho en el medievo y la modernidad, naturalmente también en el ámbito científico, es ineludible, y ha sido la base de una disputa, en ocasiones enconada, entre ciertos medievalistas y estudiosos dedicados a los inicios de la Edad Moderna.

Como es bien sabido, a lo largo del siglo xvir se extendió la idea, ya avanzada desde el humanismo, de que la Edad Media había sido un triste período de estancamiento, si no de retroceso, para la humanidad. Francis Bacon, por poner un ejemplo, en su Novum Organum, afirmaba que las edades transcurridas entre la antigüedad y la suya propia habían sido desfavorables para las ciencias, "pues ni los árabes ni los escolásticos

1 De Lagarde, G., Le naissance de l'esprit la que au déclin du Moyen Âge, Louvain, E. Nauwelaerts, $1956-1970$ 
merecen ser mencionados, pues en los tiempos intermedios, más que aumentar el peso de la ciencia la aplastaron con una multitud de tratados»². Sin duda, durante el siglo XVIII, en lo que conocemos como la ilustración, esta consideración de los hechos se torno común, e incluso se acentuó. Ejemplos podemos encontrar por doquier, si bien resulta muy significativo el caso de Condorcet, para quien «el triunfo del cristianismo fue la señal para la completa decadencia de la filosofía y de las ciencias» ${ }^{3}$.

Estos planteamientos no fueron sino reforzados durante el siglo XIX, y así, el Dictionnaire général des lettres, una enciclopedia de las muchas otras de finales del XIX (1872), se expresaba del siguiente modo: «Las artes y las letras, que parecían haber perecido en el mismo naufragio que la sociedad romana, parecieron florecer de nuevo y, después de diez siglos de tinieblas, brillar con un nuevo esplendor» ${ }^{4}$.

Estas son las ideas que estaban a la base del gran trabajo llevado a cabo por el historiador suizo Jacob Burckhardt, a quien suele atribuirse el concepto de renacimiento en su acepción moderna, y que en 1860 publicaba una obra enormemente influyente: La cultura del renacimiento en Italia. Uno de sus primeros seguidores, John Addington Symonds, escribía lo siguiente: «El renacimiento fue la liberación de la razón del calabozo, el doble descubrimiento del mundo exterior e interior. Un acontecimiento externo determinaba la dirección que tomaría este estallido del espíritu de libertad. Fue el contacto de la mente moderna con la antigua. [...] El genio moderno sintió confianza en sus propias energías cuando supo lo que los antiguos habían conseguido» ${ }^{5}$.

Así las cosas, no cabría sino considerar como triste el interés de quien dedica sus esfuerzos y su tiempo a conocer lo que, en el terreno de la ciencia, se hizo durante la Edad Media; ese período presentado como lamentable para la humanidad.

Esto no podía quedar así. En los primeros años del siglo pasado, el físico y filósofo Pierre Duhem propuso una visión radicalmente distinta de lo acontecido en la historia de la ciencia. Tras el estudio de las obras de matemáticos y filósofos de la naturaleza medievales concluyó que «la mecánica y la física de la que los tiempos modernos están justificadamente orgullosos procede, por una serie ininterrumpida de mejoras apenas perceptibles, de las doctrinas profesadas en el corazón de las escuelas medievales» ${ }^{6}$. Según su revolucionario planteamiento la interacción entre teología cristiana y filosofía natural escolástica, en las universidades medievales, era una clave sin la que no era posible comprender adecuadamente la evolución del pensamiento científico.

El debate sobre la continuidad ha presidido desde entonces el qué hacer de los investigadores durante el siglo xx y lo que llevamos del xxi. Importantes medievalistas como Charles Homer Haskins o Lynn Thorndike, en los años 20 y 30 del siglo pasado, siguieron la brecha abierta por Duhem. Y tras la segunda guerra mundial se avanzó decisivamente en la investigación histórica de la ciencia medieval, ámbito en el que destacan figuras como Marshall Claget o Anneliese Maier.

2 Bacon, F., New Organon, en Works, Nueva York, Hurd \&Houghton, 1870-1872, vol 4, p. 77.

3 Nicolas de Caritat, J. A., Marques de Condorcet, Bosquejo de un cuadro histórico de los progresos del espíritu humano, Madrid, Editora Nacional, 1980.

4 Citado por Régine Pernoud, Para acabar con la Edad Media, Palma de Mallorca, Olañeta, 1998, p. 17.

5 Citado por David C., Lindberg, Los inicios de la ciencia occidental: la tradición científica europea en el contexto filosófico, religioso e institucional (desde el 600a.cr. hasta 1450), Barcelona, Paidós, 2002, pp. 447-8.

6 Duhem, P., Les origines de la Statique, Académie de Nancy-Metz, www.ac-nancy-metz.fr/enseign/.../ Origines_de_la_statiqueTome1.doc, p.3. 
De nuevo se avivó la polémica con Alistair Crombie. Su tesis fundamental era la de que la característica clave de la ciencia moderna inicial era la posesión de un método adecuado para la práctica de la ciencia, cuya clave era la experimentación, y que este método era una creación de la Edad Media tardía; en concreto de Roberto Grosseteste. En su obra Robert Grosseteste and the origins of experimental science 1100-1700, se puede leer lo siguiente: «La tesis de este libro es que una teoría sistemática de la ciencia experimental fue comprendida y aplicada por suficientes filósofos [de los siglos XIII y XIV] para que su trabajo produjera la revolución metodológica a la que la ciencia moderna debe su origen [...] Lo que parece ser la primera aparición de una comprensión clara de los principios de la moderna ciencia experimental se encuentra en los escritos del lógico inglés, filósofo natural y estudioso Robert Grosseteste» ${ }^{7}$.

Naturalmente hubo respuesta por parte de los estudiosos de los inicios de la ciencia moderna, el más destacado de los cuales era Alexandre Koyré. En su opinión, y no nos sonará a nueva, la «revolución científica», término muy significativo en sí, de los siglos XVI y XVII no fue, como otros pretendían, un desarrollo de la ciencia medieval, sino un cambio de mentalidad que suponía la ruptura con la visión del mundo medieval. Y, así, en su «The origins of modern science. A new interpretatión», afirmaba que "Lo que los fundadores de la ciencia moderna [...] tenían que hacer no era criticar y combatir ciertas teorías erróneas, y corregirlas o reemplazarlas por otras mejores. Tenían que hacer algo totalmente diferente. Tenían que destruir un mundo y reemplazarlo por otro. Tenían que configurar de nuevo el marco de nuestro propio intelecto, reafirmar y reformar sus conceptos, desarrollar una nueva aproximación al ser, un nuevo concepto del conocimiento, un nuevo concepto de ciencia ${ }^{8}$. El hecho es que lo afirmado por Koyré, sin duda, se correspondía con la idea que de sí mismos trasmitían los hombres del siglo XVII, lo que no necesariamente tenía que ajustarse a la verdad.

Con todo, los planteamientos de Koyré influyeron poderosamente y fueron seguidos por importantes historiadores, como es el caso de A. Rupert Hall y su ensayo titulado «On the historical singularity of the scientific revolution of the seventeenth century».

La polémica no se ha cerrado, tal vez se haya refinado, y en ella han participado autores como Ernan McMullin, Medieval and modern science: continuity or discontinuity?; Thomas S. Kuhn, The estructure of scientific revolutions; o W. A. Wallace, Mechanics from Bradwardine to Galileo. Siendo ejemplos de esta disputa y de este trabajo de investigación también autores como Edith D. Sylla, John E. Murdoch, Edwart Grant, George Molland, J. A. Weisheipl..., entre otros.

No es mi intención adentrarme en esta polémica y enfocar este estudio bajo esa perspectiva. Cierto es que no considero adecuada una comprensión de la historia según la cual, en determinados momentos, la humanidad da saltos en el vacío avanzando sobre la nada. Pero el asunto de las relaciones entre la ciencia medieval y la moderna es muy complejo, y tratarlo con el rigor exigido me impediría poder hablar suficientemente, como es mi intención, sobre la labor científica de los hombres de los siglos XIII y XIV.

Es indiscutible que al final de la Edad Media, como ya señalé, se produjeron una serie de progresos, avances, novedades, en distintos ámbitos que merecen ser, cuando menos, reseñadas. Y ejemplos muy llamativos son el reloj mecánico, la pólvora y su uso o las gafas.

7 Crombie, A. C., Robert Grosseteste and the origins of Experimental Science, 1100-1700, Oxford, Clarendon Press, 1953, pp. 9-10. 13-14, 19.

8 Koyré, A., «The Origins of Modern Science: A New Interpretation», Diogenes 4(16), 1956, pp. 
Es a finales del siglo XIII cuando comienzan a construirse los primeros relojes mecánicos, y esto no es algo baladí. El tiempo se traducía en unidades de espacio sobre una esfera, se generalizó la división de la hora en 60 minutos y del minuto en 60 segundos, y así se asumía un tiempo abstracto, matemático, de unidades sobre una escala, propio de nuestro mundo y de la ciencia moderna.

Por otra parte sabemos que Roger Bacon ya mencionaba un polvo explosivo y señalaba que su potencia podía ser aumentada encerrándolo en un instrumento hecho de un material resistente. La receta más antigua, en el occidente latino medieval, que se conoce de este polvo que conocemos como pólvora, se encuentra en un manuscrito latino de alrededor de 1300, atribuido a un tal Marcos el Griego. Y el hecho es que, poco después Europa sobrepasó sobradamente a la China en la fabricación de las que llamamos armas de fuego (la ilustración más antigua de un cañón data de 1327).

También fue Roger Bacon, entre 1266 y 1267, quien, sin duda como resultado de sus investigaciones en el campo de la óptica, propuso el uso de lentes convexas para compensar la presbicia. Las lentes ya se conocían desde antiguo, pero esta propuesta fue un gran progreso. Ya a finales del siglo xiI hay pruebas de que se usaban, su fabricación estuvo en un principio asociada a la industria veneciana del cristal, y a lo largo del siglo XIV las gafas se generalizaron. Las lentes cóncavas para la miopía aún tendrían que esperar hasta el siglo xvI.

Junto a estas importantes innovaciones podrían indicarse otras tantas en distintos campos, y de ellos, al menos quiero señalar algunas que creo son interesantes en medicina o en matemáticas.

Progresos importantes tuvieron lugar en la matemática occidental desde principios del siglo XIII. Primero en los campos de la aritmética y el álgebra; y en esto jugaron un relevante papel dos pensadores de gran creatividad: Leonardo Fibonacci de Pisa y Jordano Nemorarius. Segundo en geometría, con Campanus de Novara, cuya edición de los Elementos de Euclides, de 1252, en la que se incluía el estudio de varios problemas matemáticos, siguió siendo un manual clásico hasta el siglo Xvi ${ }^{9}$.

En el siglo XIv, se desarrolló la trigonometría, cuyos iniciadores en occidente se considera que fueron John Maudit y Richard de Wallingford, diseñador de un extraordinario reloj astronómico ${ }^{10}$; contemporáneos en sus propuestas de la hecha, a este respecto, por Levi ben Gerson (1288-1344), que escribió un importante tratado sobre esta cuestión en hebreo, y que fue traducido al latín en $1342^{11}$. Se elaboró, así mismo, una novedosa teoría sobre las proporciones de la mano de Thomas Bradwardine, de quien,

\footnotetext{
9 Sobre estos autores hay mucho escrito, tan sólo citaré las obras de consulta que me han parecido de mayor interés y sencillez: Crombie, A. C., Historia de la ciencia: de San Agustín a Galileo, 2 vols., Madrid, Alianza Universidad, 1979; LindBerg, D. C., Los inicios de la ciencia occidental: La tradición científica europea en el contexto filosófico, religioso e institucional (desde el 600 a, Cr. Hasta 1450), Barcelona, Paidós, 2002; Boyer, C. B. y Merzbach, U. C., A History of Mathematics, Hoboken, New Yersey, John Wiley and sons, 2011; Moreno Castillo, P., Fibonacci: el primer matemático medieval, Madrid, Nívola, 2004; PUIG, L., "El De numeris datis de Jordanus Nemorarius como sistema matemático de signos», Mathesis, vol 10, 1994, pp. 47-92; HøYRuP, J., «Jordanus de Nemore, 13th century mathematical innovator: an essay on intelectual context, achievement, and faliure», Archive for History of exact sciences, 38, 1988, pp. 307-363; Paradís, J. y Malet, A., La génesis del álgebra simbólica, Vol. 1º, Los orígenes del álgebra: de los árabes al renacimiento, Barcelona, PPU, 1989.

10 North, J., God's Clockmaker Richard of Wallingford and the invention of Time, London, Hambledon and London, 2005.

11 Simonson, S., The mathematics of Levi ben Gerson, The Ralbag, Barllan University Press, 2000; del mismo autor: "Mathematical gems of Levi Gerson, Mathematics Teacher, 93 (8), 2000, pp. 659-663; N. L. Rabinovitch, "Rabbi ben Gerson and the origins of mathematical induction, Archive of History of Exact Sciences, 6, 1970, pp. 257-248.
} 
junto con sus sucesores del Merton College, en Oxford, tendremos que hablar mucho más detenidamente más adelante ${ }^{12}$; y Nicolás de Oresme, en París, propuso el empleo de coordenadas y gráficas para presentar la forma de una función ${ }^{13}$.

También en estos siglos se introdujeron mejoras importantes en los métodos de cálculo en el sistema de numerales hindú, como son los modernos métodos de multiplicación y división. Y curiosamente, en este tiempo, en Italia, se inventó también el libro de cuentas con el sistema de doble entrada, y en los libros de aritmética se planteaban problemas relacionados con el cambio, el interés simple o compuesto, o el descuento.

Respecto de la medicina, campo amplísimo y nada fácil de abordar, considero de especial interés señalar al menos un punto. El siglo xiv sufrió de un modo aterrador el azote de la peste negra y, de entre las consecuencias que esto tuvo en el campo de la medicina, es especialmente interesante señalar que la primera comisión de higiene pública fue organizada en 1343 en Venecia, y en 1348 en Luca, Florencia, Perugia, Pistoya y otras ciudades se dieron leyes para impedir que personas o mercancías infectadas entraran en ellas. En esta época, en Avignon, en Milán, se llevan a cabo los primeros esfuerzos sistemáticos por aislar a los portadores de la epidemia. En 1377, Ragusa promulgó una nueva ley que ordenaba el aislamiento durante treinta días de todos los viajeros procedentes de regiones contaminadas; y Marsella, en 1383, alargó este período a cuarenta días para los barcos que entraban en el puerto (cuarentena).

Todos estos hechos, tan comunes en nuestra civilización actual, manifiestan que hablamos de un período rico y lleno de actividad desde el punto de vista de la intelectualidad en general y la ciencia en particular. Pero no son sino síntomas. Debemos profundizar más, preguntarnos por qué es lo que define realmente al pensamiento científico del final del medievo, cuáles son los problemas que ocuparon a los pensadores del momento, cómo los afrontaron, qué asuntos enfrentaron a los filósofos...

Debemos tener muy presente que un hecho marca de un modo profundo a esta época. A partir, fundamentalmente, del siglo xII, el mundo occidental latino comienza a disponer de un conjunto de obras provenientes del mundo griego y del islámico, tanto científicas como filosóficas en un amplio sentido, de las que hasta entonces no se disponía.

Tratados de matemáticas, astronomía, óptica, meteorología o medicina, como los Elementos de Euclides, el Almagesto de Ptolomeo, el álgebra de al-jwarizmi, la óptica de Ibn al-haytan, o el Canon de medicina de Avicena, fueron recibidos con entusiasmo incondicional. Si bien es cierto que, como es bien sabido, lo que caracteriza al periodo que me ocupa fue el profundo impacto que ocasionó la lectura y el estudio de las obras de Aristóteles y de sus comentadores, fundamentalmente los musulmanes (al-kindi, Avicena, Algazel, Averroes), hasta entonces no traducidas.

La existencia de este nuevo saber al alcance de los hombres dedicados al estudio fue la característica central de la vida intelectual de los pensadores de los siglos XIII y XIV.

12 Verdú Berganza, I., «Thomas Bradwardine y la ciencia del siglo XIV», Zaragoza, Actas del I congreso de Filosofía Medieval, 1992, pp. 499-506.

13 Mucho se ha escrito sobre Nicolás de Oresme, cito tan sólo alguno de los trabajos que puede resultar de mayor interés: Claget, M., Nicole Oresme and the Medieval Geometry of Qualities and Motions, Madison, University of Wisconsin Press, 1968; De Oresme, N. y Grant, E., Nicole Oresme and the Kinematics of circular motion, "Tractatus de commensurabilitate vel incommensurabilitate mottum celi», Madison, London: Univ. Of Wisconsin Press, 1971; González Urbaneja, P. M., Los orígenes de la geometría analítica, Orotava, Fundación canaria Orotava de Historia de la Ciencia, 2003; Quillet, J. (Edit), Autour de Nicole Oresme, París, Vrin, 1990; Ramírez Cruz, J. A., «reflexiones sobre las ideas de Nicolás de Oresme», Asclepio, Revista de historia de la medicina y de la ciencia, 2007, Vol. LIX, nº1, Enero-Julio, pp. 23-34. 
Con el acceso al texto aristotélico completo, y al de sus grandes comentadores (recordemos que a Averroes se le conocerá en este periodo de la historia como el Comentador), los filósofos del occidente cristiano disponían de un poderoso arsenal con el que abordar, de un modo diferente, más eficiente, el estudio del universo; su comprensión.

Los nuevos textos eran extremadamente atractivos, no sólo por novedosos, sino además porque abordaban todas las cuestiones con conceptos muy potentes y fecundos. Las categorías, materia, forma, acto y potencia, las cuatro causas, en especial la formal y la final, los cuatro elementos, los contrarios, el concepto de naturaleza, la diferencia entre cualidad y cantidad, los conceptos de tiempo y espacio,..., el modo en que el aristotelismo abordaba todas estas cuestiones, y otras como el infinito, el vacío, el valor de la deducción en la ciencia,... proporcionaba un marco conceptual sumamente persuasivo con el que enfrentarse al mundo.

Junto a todo lo dicho, en las obras de psicología Aristóteles abordaba de un modo exhaustivo y profundo el alma y sus facultades, estudiando, desde una nueva perspectiva, tanto la percepción sensible como la memoria, la imaginación y el conocer intelectual mismo. Y aún más, el aristotelismo aportaba una cosmología en la que se describía el universo de un modo consistente y eran explicadas el conjunto de sus operaciones, desde el más remoto cielo hasta su centro, la Tierra. Daba una explicación del movimiento y de los fenómenos meteorológicos que iban más allá de lo hasta entonces disponible, y ofrecía un corpus biológico asombroso por su amplitud y por el detalle de sus descripciones y explicaciones.

Lógicamente, como ya había pasado en el siglo XII con el paso de la logica vetus a la logica nova, estos tesoros no podían ser despreciados. El mero hecho de que fuesen traducidos manifiesta claramente que se reconocía su valor y era creciente el interés por poder disfrutar de su estudio. Ahora se trataba de asimilar este aluvión de nuevos conocimientos, organizarlo, determinar sus implicaciones, sus aplicaciones, descubrir su sentido último... El hecho es que el aristotelismo ocuparía el centro de la escena desde entonces hasta el siglo XVII.

Sin embargo, como es bien sabido, la recepción del pensamiento aristotélico provocó encendidas polémicas que marcaron la vida intelectual del final del medievo. El nuevo material contenía una concepción del mundo que, desde el punto de vista teológico, podía suscitar problemas. Y, como veremos, una crítica puramente teórica de algunos de los principios fundamentales de la ciencia aristotélica comenzó a tener lugar, desde dentro del ya aceptado aristotelismo, en el período que ahora nos ocupa.

El interés suscitado por este conjunto de textos novedosos hizo que ya a principios del siglo xiII el aristotelismo fuese objeto de preocupación. En 1210 se prohibía en París la lectura pública o privada de las obras naturales de Aristóteles y sus comentadores. Pero el afán por el saber y el atractivo de estos textos hizo que en 1215 se tuviese que renovar el decreto, y que el Papa Gregorio IX, tras renovar la prohibición en 1228, optase, en 1231, por promover una comisión, que no llegó a reunirse, para analizar la obra de Aristóteles, de modo que pudiese ser estudiada y aprovechada sin peligro ${ }^{14}$. Al fin y al cabo la filosofía aristotélica no llenaba un vacío intelectual, sino que invadía un territorio ocupado.

Según Gregorio: «Puesto que las otras ciencias deberían servir a la sabiduría de las Sagradas Escrituras, los fieles deben apropiárselas en la medida en que se sepa que se

14 Sobre las condenas aristotélicas en el final de la Edad Media se han llevado a cabo numerosas investigaciones, y la mayor parte de ellas han quedado recogidas en la obra, coordinada por Francisco León Florido: Las condenas de Aristóteles en la Edad Media Latina, Valencia, Kyrios, 2013. 
conforman a la voluntad del dador», y añadía que puesto que «los libros sobre filosofía natural que fueron prohibidos en un consejo provincial en París [...] contienen tanto material útil como inútil», decidía, «con el fin de que lo útil no sea contaminado por lo inútil» nombrar la citada comisión que «eliminara todo lo que es erróneo o que puede causar escándalo u ofender a los lectores, de modo que cuando las materias dudosas hayan sido eliminadas, el resto pueda ser estudiado sin demora y sin ofensa» ${ }^{15}$.

Con el permiso de Oxford, cuya importancia será crucial en el siglo XIV, en el siglo XIII la Universidad de París se convirtió en el centro intelectual de la cristiandad. Y los papas eran muy conscientes de ello. En 1245 Inocencio IV se refería a ella del siguiente modo: «París es el crisol en el que el oro se funde, en que se ha construido la Torre de David fortificada con murallas [...], de ella se ve salir continuamente a los más fuertes de entre los fuertes llevando sus espadas, y a hombres sabios en el arte de la guerra, que han de recorrer la tierra entera», y en 1255 Alejandro IV afirmaba: «La ciencia de las escuelas de París es en la Santa Iglesia como el árbol de la vida en el Paraíso terrenal y como la lámpara resplandeciente en la Casa del Señor. Como una madre fecunda en erudición hace brotar con toda abundancia, de sus fuentes de doctrina salvadora, los ríos que van a regar la faz estéril de la tierra; [...] Es en París donde el género humano, deformado por la ceguera de su ignorancia original, recobra su vista y belleza por el conocimiento de la verdadera luz que despide rayos de ciencia divina ${ }^{16}$.

No era extraño, por tanto, que se extremase el cuidado respecto de lo que allí se hacía. Pero, el hecho es que Aristóteles fue ganando terreno de modo inapelable. Los grandes maestros no podían dejarlo de lado, y en torno a su asimilación se crearon corrientes de pensamiento distintas.

La enseñanza de la lógica del estagirita, a la que no afectaban las prohibiciones, junto con la total disponibilidad de las obras sometidas a vigilancia (a pesar de que su enseñanza estuviese prohibida) y la paulatina recuperación de las obras de sus comentadores, en especial de Averroes, convirtieron a Aristóteles en el centro de atención, y sus obras en lectura inexcusable. Y así, en 1255 los papeles se habían invertido hasta tal punto que, la facultad de Artes pasaba a los nuevos estatutos lo que, en verdad, ya era práctica común: las clases sobre las obras conocidas de el filósofo.

Figuras de la altura intelectual de Roberto Grosseteste, Roger Bacon, S. Alberto Magno, S. Buenaventura, Santo Tomás de Aquino, Gil de Roma, Sigerio de Brabante, Boecio de Dacia, Duns Escoto o incluso Guillermo de Ockham tomaron posición al

15 «Magistris W. Archidiacono Belvacensi, Symoni de Ateis Ambianensi et St. De Pruvino Remensi canonicis. Cum Sapientie sacre pagine relique scientie debeant famulari, eatenus a fidelibus amplectende, quatenus obsequi dinoscuntur beneplacitis donantis ut si quid in eis fuerit virulentum vel aliter vitiosum, quo derogari possit fidei puritati, eminus respuatur, quia inventa in numero captivorum mulier speciosa non aliter in domum permittitur introduci, nisi rasa superfluitatis cesarie ac unguibus lacerantibus circumcisis, et ut spoliatis Egyptis ditentur Ebrei, jubentur vasa aurea et argéntea pretiosa non eruginosa enea vel lutea mutuari. Ceterum cum sicut intelleximus libri naturalium, qui Parisius in Concilio provinciali fuere prohibiti, quedam utilia et inutilia continere dicantur, ne utile per inutile vitietur, discretioni vestre, de qua plenam in Domino fiduciam obtinemus, per apostolica scripta sub ostentatione divini judici firmiter precipiendo mandamus, quatinus libros ipsos examinantes sicut convenit subtiliter et prudenter, que ibi errónea seu escandali vel offendiculi legentibus inveneritis illativa, penitus resecetis ut que sunt suspecta remotis incunctanter ac inoffense in reliquis studeatur. Dat. Laterani Viiij Kal. Maii, pontificatus nostri anno quinto", Denifle, H.-Chatelain, E., Chartularium Universitatis Parisiensis, París, Delalain, 1891, Vol. I, no 87, pp. 143-4.

16 GILson, E., La filosofía en la Edad Media; desde los orígenes patrísticos hasta el fin del siglo XIV, Madrid, Gredos, 1982, pp. 370-1. 
respecto, y, como bien sabemos, en algunos casos hicieron del aristotelismo la base de su pensamiento.

Con todo, no debemos olvidar que este extraordinario mundo que se abría ante los ojos del hombre del siglo xiII era un mundo no cristiano. Constituía una tarea sumamente delicada su encaje en una cosmovisión cristiana, y pronto se convirtió en un formidable motivo de controversia. Incluso hubo pensadores que, llevados de su entusiasmo, comenzaban a mantener posiciones difícilmente conciliables con la visión del mundo que hundía sus raíces en el credo cristiano, y parecían defender, entre otras cosas, que el filósofo, fiel seguidor de Aristóteles, podía alcanzar por sí mismo, usando adecuadamente su razón, todas las verdades y la felicidad misma. «Pertenece al filósofo [decía Boecio de Dacia] determinar cualquier cuestión que pueda ser discutida por la razón; pues toda cuestión que pueda ser discutida mediante argumentos racionales cae dentro de alguna parte del ser. Pero el filósofo investiga todo el ser: natural, mecánico y divino. Por tanto, pertenece al filósofo determinar toda cuestión que pueda ser discutida mediante argumentos racionales» ${ }^{17}$.

Así las cosas, el obispo de París Esteban Tempier condenaba, en 1270, trece tesis, supuestamente mantenidas por maestros parisinos, y en 1277, con el impulso papal, extendía la condena a 219 , definidas como «errores manifiestos y execrables» que «algunos hombres de estudio en artes, sobrepasando los límites de su propia facultad, osan exponer y disputar en las escuelas, como si fuera posible dudar de su falsedad» ${ }^{18}$.

Esta archiconocida condena, que tuvo su eco en Oxford en el mismo año de la mano de Robert Kilwardby y en 1284 de la de John Pecham ${ }^{19}$, fue extraordinariamente importante, y marcó el devenir del pensamiento. Tan es así que, para Pierre Duhem constituyó el acta de nacimiento de la ciencia moderna; afirmación muy atrevida cuyos posibles fundamentos veremos más adelante. E importantes pensadores del siglo XIV, como Thomas Bradwardine, en el Oxford de la primera mitad del siglo, o Nicolás de Oresme, en el París de la segunda, la tenían presente como autoridad al proponer sus ideas.

La condena iba dirigida, fundamentalmente, contra todo lo que ponía en entre dicho la libertad, en Dios y en el hombre, así como la omnipotencia del primero. Se reconocía un peligroso determinismo en el aristotelismo pujante. Y lo paradójico es que, así, a la vez que Aristóteles se imponía como referencia fundamental en el ámbito universitario, importantes principios de su filosofía natural empezaban a cuestionarse.

El aristotelismo, a grandes rasgos, concebía un universo finito, cerrado, estructurado de acuerdo con relaciones necesarias, conocidas gracias a la metafísica y demostrables gracias a la lógica. De forma esférica, estaba dividido en dos regiones distintas, hechas de distinto material y que operaban según distintos principios. La región sublunar (la Terrestre), constituida por los 4 elementos, era el lugar de la generación y la corrupción y de los movimientos transitorios, en los que cada elemento se dirige hacia su lugar natural. La región supralunar, donde se sitúan las estrellas fijas, el sol y los demás

17 LindBerg, D. C., Los inicios de la ciencia occidental. La tradición científica europea en el contexto filosófico, religioso e institucional (desde el 600 hasta 1450), Barcelona, Paidós, 2002, p. 299.

18 «Magnarum et gravim personarum creba zeloque fidei accensa insinuavit relatio, quod nonnulli parisius studentes in artibus propriae facultatis limites excedentes quosdam manifestos et exsecrabiles errores, immo potius vanitates et insanias falsas, in rotulo seu cedulis praesentibus hiis annexo seu annexis contentos, quasi dubitabiles in scholis tractare et disputare praesumunt...», H. Denifle, E. Chatelain, Chartularium Universitatis Parisiensis, Vol. I, París, Delalain, 1889, pp. 543-558.

19 Sobre Robert Kilwardby es interesante LagerLund, H. y Tном, P. (Eds.), A companion to the philosophy of Robert Kilwardby, Leiden, Brill, 2012. Y sobre John Pechkam es sumamente interesante la lectura de sus cartas como arzobispo de Canterbury, dirigidas a la Universidad de Oxford y al Obispo de Lincoln: C. Martin, Registrum epistolarum fratris Joannis Pechkam, III, Londres, 1885, pp. 896-902. 
planetas, estaba constituida por esferas celestes concéntricas, y se caracterizaba por su perfección inmutable y el movimiento circular uniforme. Así mismo, consideraba que los movimientos celestes causaban la generación y la corrupción en la región terrestre.

Ciertos elementos de esta cosmología se convirtieron en objeto de crítica y debate, ampliándose finalmente a cuestiones relativas al papel de la matemática, la explicación del movimiento, el valor de la inducción... Y es esta labor de reevaluación del aristotelismo la que propició la mayor contribución al ámbito de la ciencia por parte de los investigadores medievales.

Los artículos del Syllabus ${ }^{20}$ que recalcaban el infinito poder creador de Dios motivaron todo tipo de especulaciones sobre mundos y situaciones imaginarias que, indiscutiblemente, Dios podía crear. Esto provocó el desarrollo de una filosofía natural especulativa o hipotética durante el siglo XIV; siglo en el que los principios de la filosofía de la naturaleza aristotélica fueron clarificados, criticados e incluso rechazados. Veamos algunos ejemplos más despacio.

Aristóteles había negado explícitamente la posibilidad del espacio, lugar o vacío fuera del mundo, y esto era admitido hasta que la condena de 1277 provocó una revisión del asunto. De hecho el artículo 49 condenaba la tesis según la cual «Dios no podría mover el cielo con un movimiento rectilíneo. Y la razón es que entonces dejaría tras de sí el vacío ${ }^{21}$. La mayor parte de los pensadores se limitaron a aceptar que Dios, omnipotente, pudo crear un espacio vacío fuera del mundo, pero unos pocos, como Thomas Bradwardine o Nicolás Oresme afirmaron que realmente lo había hecho, llegando el primero a identificar este espacio vacío con la omnipresencia divina y a afirmar que, puesto que Dios es infinito, el espacio vacío extracósmico debe igualmente ser infinito. Oresme sacó importantes consecuencias de esta idea, y no pocos investigadores han señalado la posible relación de estas ideas con las que siglos después expondrá Newton.

Se discutió el concepto de continuo y de infinito, de lo que fue un ejemplo el Tractatus de continuo de Thomas Bradwardine ${ }^{22}$, así como problemas como el de la máxima resistencia que una fuerza podía vencer y la mínima que no podía superar, adelantando intuiciones que sólo mucho más tarde tendrían adecuado desarrollo y poniendo las bases lógicas del cálculo infinitesimal.

No menos interesante es la propuesta que plantearon Buridán y Oresme, según la cual era posible que, contraviniendo a Aristóteles, no fuesen las esferas celestes las que rotasen alrededor de la tierra, sino la Tierra la que rotase sobre su eje. No era una idea por completo nueva, pero los planteamientos sí.

La intención no era quitar a la Tierra del centro del cosmos, pero si discutir su inmovilidad. Y quien discutió la cuestión de un modo más ingenioso y profundo fue Oresme en su Livre du ciel et du monde, un comentario al De caelo de Aristóteles escrito en 1377 por encargo del rey Carlos V de Francia ${ }^{23}$.

20 «Syllabus» es el nombre con el que se conoce a las condenas promovidas por Esteban Tempier en 1277 .

21 «Quod Deus non possit movere caelum motu recto. Et est ratio, quia tunc relinqueret vacuum», F. Leon Florido, 1277; la condena de la filosofía: estudio y traducción del Syllabus de Esteban Tempier, Madrid, A parte Rei, Revista de Filosofía, (66), 2007, pp. 65-107.

22 Murdoch, J. E., Geometry and the continuum in the fourteenth century: a philosophical analysis of Thomas Bradwardine's Tractatus de continuo, Madison, University of Wisconsin, 1957; E. Wilks Dolnikowski, Thomas Bradwardine: a view of Time and Vision of Eternity in Fourteenth century Thought, Leiden, Nueva York, Colonia, Brill, 1995.

23 Oresme, N., Livre du ciel et du monde, (Eds. Denomy, A. J. y Menut, A. D. ), Madison, University of Wisconsin press, 1968. 
En el capítulo 25 del $2^{\circ}$ libro afirmaba que le parecía posible defender la opinión, siempre sujeta a corrección, de que la Tierra se mueve con movimiento diario y los cielos no. Y no sólo eso, sino que además era imposible demostrar lo contrario por ninguna observación, o por la razón, proponiéndose, frente a las objeciones, aportar razones en favor de esta opinión.

La primera objeción consistía en que lo que vemos es que se mueven las esferas celestes, los planetas, no la tierra. A lo que contestaba afirmando que todo lo que percibimos es movimiento relativo, y que por tanto no era la observación la que podía zanjar el asunto, pues los cálculos astronómicos no se verían afectados; «todas las conjunciones, oposiciones, constelaciones, figuras e influencias de los cielos serían justamente como son en todos los sentidos..., y las tablas de los movimientos y todos los otros libros serían tan verdaderos como lo son ahora, excepto solamente que se diría que el movimiento diario es aparente en los cielos y real en la Tierra» ${ }^{24}$.

La segunda objeción a partir de la experiencia consistía en que si la Tierra giraba por el aire de oeste a este habría u soplo de viento fuerte y continuo de este a oeste. A lo que replicaba que tanto el agua como el aire participaban de la rotación de la Tierra, y por tanto no habría el viento indicado.

La tercera de las objeciones había sido planteada por Buridan. La idea era que una flecha lanzada verticalmente hacia arriba, en ausencia de viento, en una tierra rotatoria no volvería a su punto de partida, ya que, mientras surcaba el aire, la tierra se habría movido. Y puesto que no es eso lo que ocurre, por reducción al absurdo, hay que afirmar que la Tierra no rota. Oresme replicó haciendo ver que, sobre una tierra rotatoria, mientras la flecha se mueve verticalmente hacia arriba y hacia abajo, también se mueve horizontalmente a la vez que la tierra, y por tanto, lo objetado por Buridan no sucedería. Y para reforzar su argumento, propuso un ejemplo, experimento mental, a bordo de una nave, muy parecido al que usó siglos después Galileo: «Esto parece ser posible de este modo, pues si un hombre fuera en un barco que se moviera muy rápidamente hacia el este sin que él fuera consciente de dicho movimiento, y si moviera sus manos en una línea recta hacia abajo a lo largo del mástil del barco, a él le parecería que su mano se está moviendo con un movimiento rectilíneo. Ahora bien, de acuerdo con esta opinión a nosotros nos parece que sucede lo mismo con la flecha que es disparada recto hacia abajo o hacia arriba. Dentro de la nave que se mueve así, puede haber toda clase de movimientos [...] y nos parece que son exactamente los mismos que los que se producen cuando la nave está parada. Así pues, si un hombre en esta nave caminara hacia el oeste menos rápidamente de lo que la nave se está moviendo hacia el este, a él le parecería que se está moviendo hacia el oeste, cuando realmente se mueve hacia el este. Y, de modo similar al caso precedente, todos los movimientos aquí abajo parecerían los mismos que si la Tierra estuviera quieta ${ }^{25}$.

Por último, y no es menos significativo, argumentaba que los pasajes de las Escrituras que parecían enseñar la fijeza de la Tierra podían entenderse como un modo en que el lenguaje del texto bíblico «se adecúa al uso común del lenguaje popular» ${ }^{26}$.

Los principales argumentos positivos que Oresme adujo a favor de la rotación de la Tierra sobre su eje se centraban todos en que era una solución más sencilla. Con todo, al

24 Sobre este asunto merece la pena leer la obra ya citada de CRomBIE, A. C., Historia de la ciencia: de San Agustín a Galileo, 2 Vols, Madrid, Alianza Editorial, 1987, Vol. 2, pp. 74-82, (en concreto, el texto transcrito es citado en las páginas 81 y 82).

25 Ibidem.

26 Ibid. 
final ni tan siquiera él se adhirió a esta hipótesis, que, como sabemos, hubo de esperar para ser adoptada.

En astronomía la autoridad aristotélica se vio contestada por la teoría de los epiciclos y las excéntricas de Ptolomeo, que se impuso durante la época tardomedieval al modelo de Eudoxo, que era el gran propagandista de la cosmología aristotélica. Los astrónomos, que exigían resultados cuantitativos, encontraban respuestas en los modelos Ptolemaicos, y los maestros de las universidades elaboraron pronto sus propios libros. Es el caso de Juan Sacrobosco (John Holywood) y su obra titulada La esfera, escrito a mediados del siglo xIII y utilizado como manual aún en el siglo XVII, o el Theorica Planetarum, escrito poco más tarde por un autor anónimo y al que frecuentemente se adjuntaban las Tablas alfonsinas, elaboradas en la corte de Alfonso X de Castilla ${ }^{27}$.

Otros campos, de gran interés, en los que los hombres de ciencia del XIII y el xIV se vieron en la necesidad de repensar a Aristóteles fueron los de la relación entre cualidad y cantidad, el valor de las matemáticas en la ciencia de la naturaleza, es decir, en la física, y la adecuada comprensión y explicación del cambio, y, en especial el de lugar, es decir, el movimiento.

Podría decirse que una de las grandes contribuciones de este período a la constitución de un nuevo método científico fue la extensión de la matemática a todo el campo de la ciencia física, al menos en principio.

Aristóteles restringía el empleo de las matemáticas, de acuerdo con su idea de la subordinación de unas ciencias a otras, distinguiendo con claridad los papeles de la matemática y de la ciencia que realmente se ocupaba de la naturaleza, la física. Los científicos de finales del medievo comenzaron a mostrar menos interés por la pregunta metafísica o, en términos aristotélicos, física de la causa, y plantearon preguntas que respondían con una teoría matemática. Ejemplos claros de esto se encuentran ya en el siglo XIII en los campos de la óptica o la astronomía, y será en el xiv cuando se manifieste este cambio de mentalidad de un modo más interesante.

Esta convicción podemos encontrarla en textos de personalidades claves del siglo XIII como Roberto Grosseteste o Roger Bacon, cuyo trabajo, entre otros campos, en el de la ciencia, fue extraordinario. "Sólo en las matemáticas existe ciencia y demostración en sentido estricto», decía el primero en su Comentario a los Analíticos posteriores, y afirmaba en sus obras De natura locorum y De lineis: «A partir de las reglas y principios y fundamentos [...] dados por el poder de la geometría, el observador cuidadoso de las cosas naturales puede dar la causa de todos los efectos naturales», «Es de la mayor utilidad el considerar las líneas, los ángulos y las figuras porque es imposible entender la filosofía de la naturaleza sin ellos [...] Porque todas las causas de efectos naturales han

27 Goddu, A., Copernicus and the aristotelian tradition: education, Reading and philosophy in Copernicus path to heliocentrism, Leiden, Boston, Brill, 2010, pp. 147-151; Campano de Novara, Campanus of Novara and Medieval planetary theory: Theorica Planetarum, (Edición, Traducción e introducción al inglés de F. S. Benjamin Jr. Y G. J. Toomer), Madison, University of Wisconsin Press, 1971; Juan Sacrobosco, The Sphere of Sacrobosco and its comentators, (Edición y traducción a cargo de L. Thorndike), Chicago, Chicago University Press, 1949; Mccluskey, S. C., Astronomies and Cultures in Early Medieval Europe, Cambridge, Cambridge University Press, 2000, pp. 192-209; Pedersen, O., «In quest of Sacrobosco», Journal for the History of Astronomy, 16, 1985, pp. 175-221; KNORR, W. , "Sacronbosco's Quadrant: date and sources», Journal for the History of Astronomy, 28, 1983, pp. 187-222; BonMATI SÁnCHEz, V. , «La revolución científica del siglo xvi; de la Sphaera mundi de Juan de Sacrobosco al De Revolutionibus de Nicolás Copérnico", en Humanismo y pervivencia del mundo clásico: Homenaje al Profesor Antonio Fontán; Literatura Latina y Renacentista, vol. 3, 2002, pp. 1407-1412; Federici-VescovinI, G., "Michel Scot et la Theorica planetorum gerardi», Early Science and Medicine, vol. 1, n 2 (Jun.1996), pp. 272-282; Costa, A., "Las obras astronómicas en los talleres alfonsíes (I)», Ayeres, 1, 1990, pp. 4-12. 
de ser expresadas por medio de líneas, ángulos, figuras, porque de otro modo sería imposible tener conocimiento de la razón de estos efectos» ${ }^{28}$. Y en esta línea decía Bacon en su Opus Maius: «Ninguna ciencia puede ser conocida sin las matemáticas», «en las cosas de este mundo, por lo que respecta a las causas eficientes y generativas, no puede conocerse nada sin el poder de la geometría ${ }^{29}$.

Sin embargo será en el Oxford del siglo xiv donde esta perspectiva matemática de la naturaleza, de la mano de uno de los más brillantes pensadores de la época, Thomas Bradwardine, alcance su mayor expresión: «La Matemática es lo que revela más genuinamente la verdad, ayuda a conocer los muchos secretos escondidos y tiene la llave para conocer lo oculto de las palabras; quien, por tanto, tenga la desfachatez de estudiar física, rechazando la matemática descubrirá con asombro que nunca hará su entrada a través del pórtico de la sabiduría ${ }^{30}$.

Bradwardine, con su Tractatus de proportionibus o De proportione velocitatum in motibus, compuesto en 1328, y sus seguidores, Ricardo Swineshead, Guillermo Heytesvury, Juan Dumbleton, por citar a los más destacados, conocidos como los calculadores del Merton College, abordaron la tarea de analizar las variaciones de intensidad de las cualidades y los movimientos, la cuantificación de las cualidades, y alcanzaron lo que se considera, tradicionalmente, como el primer paso hacia la construcción de la moderna física matemática ${ }^{31}$.

Para Aristóteles cantidad y cualidad eran cuestiones radicalmente distintas. Así pues, el cambio cuantitativo y el cualitativo no debían confundirse, no teniendo relación entre sí. Desde este punto de vista, resultaba inoperante, ya que no reflejaba adecuadamente la realidad, explicar la cualidad acudiendo a la cantidad. Y así como se entendía que pasar de estar alegre a estar triste, o de estar en gracia a estar en pecado, eran cambios cualitativos que lejos de entenderse se desvirtuarían si se explicasen como cambios de orden cuantitativo, se consideraba que el cambio de color, el aumento o disminución del calor, de la intensidad de luz, e, incluso, el movimiento eran cambios de orden cualitativo.

Bradwardine (Doctor Profundus) y sus colegas, en acuerdo con la brecha que en el terreno de la epistemología abrió Guillermo de Ockham y con lo que en el campo de la óptica se había intentado desde Grosseteste y Witelo, rechazaron la tesis aristotélica y concluyeron que las diferencias cualitativas se debían a diferencias de carácter cuantitativo, sólo explicables y cognoscibles a través de la matemática. Y lo más importante, propusieron un método que permitía la cuantificación de las cualidades, es decir, conectar adecuadamente matemática y física.

El trabajo del Doctor Profundus se centró en el estudio del movimiento, contribuyendo al desarrollo de la cinemática mediante la definición de algunos conceptos fundamentales como son los de movimiento uniforme, aceleración uniforme, velocidad instantánea..., y haciendo análisis en términos de distancia y tiempo, dos nociones cuya combinación no contemplaba la física aristotélica, considerando que la velocidad (variable dependiente) se explica en una función algebraica de distancia y tiempo (variables independientes).

28 Citado por Crombie, A. C., Historia de la ciencia: de San Agustín a Galileo..., Vol. 2, pp. 24 y 28. Merece la pena consultar la gran obra de CRomBIE, A. C.: Robert Grosseteste and the origins of the experimental science, 1100-1700, Oxford, Clarendon Press, 2003.

${ }_{29}$ Citado por Crombie, A. C., en Historia de la ciencia..., Vol.2, p. 30.

30 Bradwardine, T., Tractatus de continuo, MS. Erfurt, Amplon. Q. 385 Fol. 31V.

31 Verdú Berganza, I., "Thomas Bradwardine y la ciencia del siglo XIV», Actas del $1^{\circ}$ congreso de Filosofía Medieval, Zaragoza, 1992, pp. 499-505. 
Además, el método utilizado, el «algebra de palabras» de Bradwardine, empleaba letras del alfabeto para sustituir a las cantidades de las variables, describiendo las operaciones con palabras; uno de los primeros intentos conscientes de introducir un formalismo algebraico. Y este modo de plantear la cuestión le permitió reformular las tesis aristotélicas sobre el movimiento violento.

En el capítulo $5^{\circ}$ del libro VII de la Fisica, Aristóteles discutía varias y distintas proporciones (relaciones proporcionales) entre lo que movía un cuerpo, la fuerza motriz (f) y la resistencia (r), y establecía dos principios: $1^{\circ}$ que no todos los movimientos locales eran comparables y $2^{\circ}$ que doblándose la fuerza o dividiéndose a la mitad la resistencia, se doblaba la velocidad. Este era un asunto que había ocupado a los grandes conocedores de la obra aristotélica, y Bradwardine, que mostró la insuficiencia de esta explicación (conducía a errores), propuso una nueva teoría sobre cómo estaban relacionadas fuerza, resistencia y velocidad en todo movimiento.

La conocida como «ley de Bradwardine» afirmaba que el cambio de velocidad (la ratio de la distancia en función del tiempo) se seguiría del cambio de la ratio completa de la fuerza en función de la resistencia, y, así, para doblar la velocidad, la ratio entera debía ser doblada (multiplicada por sí misma o elevada al cuadrado); y, del mismo modo, la mitad de la velocidad se seguiría de la medietas de la ratio, esto es, no de dividirla por dos, sino de la resolución de la raíz cuadrada. «La proporción de la velocidad en el movimiento se sigue de la proporción de la fuerza a la resistencia y viceversa o, en otras palabras: las proporciones de las fuerzas a las resistencias son proporcionales a la velocidad del movimiento y viceversa. Y lo pensado aquí es geométricamente proporcional»32. (Capítulo 3, conclusión 1).

El Tractatus de proportionibus se convirtió en un hito en la historia de la ciencia. Suponía la aplicación de una nueva y compleja teoría matemática al movimiento, capaz de justificar los hechos observables, cuantificaba una supuesta cualidad, llamada «moción dinámica», poniendo las bases de lo que fue el trabajo de los llamados «Calculadores del Merton College»; y algo muy importante, en el capítulo IV de la obra proponía la idea de que la misma ley debía explicar tanto los movimientos sublunares como los supralunares, idea que rompía con la cosmología aristotélica y habría de esperar hasta Newton para ser materializada.

El resto de los calculadores se volcaron en el estudio de las proporciones con la intención de expresar los grados en que aumenta o disminuye una cualidad respecto a una escala fijada previamente. Llamaron forma a cualquier cantidad o cualidad variable en la naturaleza y supusieron que la intensio (intensidad) de una forma era un valor numérico que se le asignaba. Del mismo modo, hablaban de la velocidad con que cambia la intensio con respecto a otra forma conocida, a la que llamaron extensio (también utilizaban los términos latitud y longitud). Así, se podía fijar la intensio de la velocidad (y la velocidad con que esta intensio cambia) por referencia a la extensio de la distancia y el tiempo.

Esta labor teórica permitió definir una serie de conceptos fundamentales como los de Movimiento uniforme, cambio disforme (aceleración), cambio uniformemente disforme (uniformemente acelerado), cambio disformemente disforme o velocidad instantánea, referidos al cambio de una intensio cualquiera.

Y llevaron a cabo desarrollos concretos, el más importante de los cuales es el conocido como «teorema de la velocidad media» o «teorema de Merton», según el cual un

32 CRosby, H. L. Jr., Thomas bradwardine. His "Tractatus de Proportionibus». Its significance for the development of mathematical physics, Madison, University of Wisconsin press, 1955, p. 112. 
cuerpo en movimiento uniformemente acelerado recorre, en un determinado intervalo de tiempo, el mismo espacio que sería recorrido por un cuerpo que se desplazara con velocidad igual a la velocidad media del primero; lo que en sus términos venía a ser que un cuerpo que inicia la aceleración uniforme a partir del reposo recorre cierta distancia en cierto tiempo y que, si el mismo cuerpo hubiera de estar en movimiento durante el mismo intervalo de tiempo con una velocidad uniforme igual a la velocidad instantánea en el instante medio de su aceleración uniforme, recorrería una distancia igual.

La influencia de lo hecho por los «Calculadores» fue formidable, siendo estudiados durante siglos en París, Padua o Salamanca, e incluso por Galileo ${ }^{33}$. Y, sin duda, merece ser citada al menos la prueba que del «teorema de Merton» propuso en la segunda mitad del siglo xiv Nicolás de Oresme en su Tractatus de configurationibus qualitatum et motuum. En esta obra recurría a la geometría, y, en concreto al uso de gráficas, en lo que suponía una extraordinaria intuición matemática sobre la que trabajarían siglos después hombres como Descartes o Fermat ${ }^{34}$.

Estos indudables avances en el terreno de la cinemática fueron acompañados por la necesidad de repensar también a Aristóteles en el campo de la dinámica tanto en Oxford, como ya hemos visto en parte, como en París, lo que dio como fruto la ingeniosa propuesta de Buridan, conocida como teoría del impetus.

La cuestión era explicar del mejor modo posible el movimiento no natural. Cuando un objeto se movía y no lo hacía dirigiéndose a su lugar natural, una fuerza motriz (motor) tenía que estar actuando sobre él para que venciese su inclinación natural. Al fin y al cabo, era un principio aristotélico que todo lo que se mueve es movido por otro, y que ese otro (el motor) es extrínseco respecto del móvil. El problema residía en explicar cuál era ese motor en el caso de una flecha cuando ésta, sin contacto ya con la cuerda que la lanzó, surca el aire.

Los comentadores de Aristóteles, durante siglos, habían discutido el asunto y propuesto soluciones. Buridan supuso que la causa del movimiento de un objeto una vez separado del motor impulsor inicial era tan solo una y se mantenía a lo largo de todo el movimiento. Esta fuerza impulsora, que llamo impetus, se transmitía del impulsor al cuerpo en movimiento, quedaba impresa en el móvil y actuaba como causa de su movimiento. Su idea era que, naturalmente, si no fuese por la acción de otras fuerzas independientes como la resistencia y la atracción que sufre el objeto por su lugar natural, el

33 Grant, E., «Bradwardine and Galileo: Equality of velocities in the void», Archive for the history of Exact Sciences, 2, 1965, pp. 344-364; Wallace, W. A., Machanics from Bradwardine to Galileo, Washington D. C., The Catholic University of America, 1971; "The Calculatores in Early Sixteenth Century Physiscs», The British Journal for the History of Science, 4, 1969, pp. 221-232; LEwIs, C. J. T., The Merton tradition and kinematics in Late Sixteenth and Early seventeenth Century Italy, Tesis Doctoral inédita, Londers, Universidad de Londres, 1975; "The fortunes of Richard Swineshead in the time of Galileo», Anals of Science, 33, 1975, pp. 561-584; SyLla, E. D., «The origin and fate of thomas Bradwardine's De proportionibus velocitatum in motibus in relation to the history of mathematics", Machanics and natural philosophy before the scientific revolution, (W. R. Laird y S. Rous Eds.), Dordrecht, Springer, pp. 67-119; Crombie, A. C., Historia de la ciencia..., Vol. 2, pp. 50-112.

34 DE Oresme, N., Tractatus de configurationibus qualitatum et motuum. [Nicole Oresme and the medieval geometry of qualities and motions. A treatise on the uniformity and difformity of intensities know as Tractatus de Configurationibus Qualitatum et Motuum], Edición, traducción al inglés, comentarios e introducción de M. Clagett, Madison, Wisconsin University Press, 1968; Albares, R., «Proporción y configuración en Nicolás de Oresme: el Tractatus de configurationibus qualitatum et motuum», Acras del Simposio: Filosofía y Ciencia en el Renacimiento, Santiago de Compostela, Servicio de Publicaciones de la Universidad de Santiago de Compostela, 1088, pp. 131-140; RoBles, J. A., «Oresme y la filosofía moderna», Mathesis. Filosofía e historia de las matemáticas, Vol. IX, nº 1, Febrero, México, UNAM, 1993, pp. 1-31. 
cuerpo permanecería en movimiento de modo indefinido, en la misma dirección y de modo constante.

Influido por Bradwardine formuló su teoría cuantitativamente, estableciendo que el impetus de un cuerpo era igual a su cantidad de materia multiplicada por la velocidad, con lo que explicaba que si un cuerpo más denso y pesado era impulsado con la misma velocidad que otro más ligero, el primero recorrería más distancia porque podía recibir más ímpetus y retenerlo más tiempo (planteando por $1^{\mathrm{a}}$ vez, si bien no en los mismos términos, el problema de la relación entre fuerza y energía que enfrentaría a Newton y Huygens).

Pero, tal vez, lo más interesante sea el hecho de que, como había hecho el Doctor profundus, propuso que, contra la opinión de Aristóteles, cualquier movimiento, natural o violento, terrestre o celeste, podía ser explicado recurriendo al ímpetus. Este era el caso del movimiento de una flecha en el aire, el de la caída de los graves, en los que era la propia Gravitas la que generaba ímpetus en cada instante en que el grave se movía, lo que hacía que con el tiempo se incrementase la velocidad, o, incluso, el caso de las esferas celestes: «Uno no encuentra en la Biblia que haya Inteligencias encargadas de comunicar a las esferas celestes sus movimientos adecuados ; está permitido, pues, demostrar que no es necesario suponer la existencia de estas Inteligencias. Se puede decir, de hecho, que Dios, cuando creó el universo, puso en movimiento las esferas como le plugo, imprimiendo a cada una de ellas un ímpetus que la ha movido desde siempre. Dios no tiene, por tanto, que mover más a estas esferas, excepto ejerciendo un influjo general parecido a ese por el que da su cooperación a todos los fenómenos. [...] Estos ímpetus que Dios imprimió en los cuerpos celestes no han sido reducidos o destruidos por el paso del tiempo, porque no había en los cuerpos celestes ninguna inclinación hacia otros movimientos y no había resistencia que pudiera corromper o retener a esos ímpetus ${ }^{35}$.

(Quaestiones super octo libros phisycorum).

Grosseteste, Bacon, Bradwardine, Buridan, Oresme..., fueron hombres extraordinariamente brillantes, pero, como sabemos, no eran excepciones en el ambiente intelectual de los siglos xIII y xIV, siglos de una riqueza asombrosa en todos los campos del pensamiento. Es difícil, por tanto, hacer justicia a estos siglos, a estos hombres y a sus aportaciones en el campo de la ciencia en unas pocas páginas. Pero sin duda cometería una grave injusticia si no me detuviera, por poco que sea, y tratase de mostrar lo que supuso para este ámbito del pensamiento Guillermo de Ockham.

Como muy bien muestra Olga L. Larre en su obra La filosofía natural de Ockham. Una fenomenología del individuo ${ }^{36}$, de las numerosas obras que Ockham dedico al estudio de la naturaleza se pueden extraer una serie de criterios referentes a la investigación científica, estrechamente ligados a su nueva lógica y a la crítica, que llevo a cabo, de la cosmología tradicional aristotélica. Aceptado, de acuerdo con lo prescrito en 1277, que el mundo es esencialmente contingente, creado por la absoluta libertad de un Dios omnipotente, no era lícito partir del supuesto aristotélico de que el mundo está estructurado de acuerdo con relaciones en sí necesarias, conocidas gracias a la metafísica. Para Ockham resultaba obvio que no sólo no era preciso admitir una multiplicidad de for-

35 Este texto se encuentra citado en Crombie, A. C., Historia de la ciencia..., vol.2, p. 69 y en A. Maier, Zwei Grundprobleme der Scholastischen Naturphilosophie, Roma, Roma: Edizioni di Storia e Letteratura, 1968, pp. 211-212. 2000 .

36 Larre, O. L., La filosofía natural de Ockham; una fenomenología del individuo, Pamplona, EUNSA, 
mas, además de los individuos, sino que no respondía a lo que implicaba la concepción de un mundo creado por un Dios omnipotente y libre. Y si esto era así, el fundamento del conocimiento científico no podía estar en el conocimiento metafísico de las formas, sino en el experimental. Y es de aquí de donde surge el primer criterio, claramente presente en la ciencia moderna: sólo se puede conocer de manera científica aquello que es controlable mediante la experiencia empírica.

Por otra parte, la radical fidelidad de Ockham a lo concreto le llevo, en un uso francamente atrevido de su famosa navaja, a rechazar toda substancialización de carácter metafísico de entidades como el movimiento, el espacio, el tiempo y el lugar natural, todo lo cual está en la base de lo que podríamos considerar como el $2^{\circ}$ criterio fundamental del método científico ockhamista, según el cual, más que preocuparnos por el qué de los fenómenos debemos dedicarnos al cómo de su acontecer.

Las propuestas de Ockham ponen de manifiesto que lo importante no es la naturaleza, en términos aristotélicos, sino la función. Se abandona la metafísica en favor de la física, en el sentido moderno del término. Y esto lo vieron con cierta claridad sus contemporáneos. Estas nociones condujeron a una matematización de la ciencia y, como hemos visto, a la aplicación de los métodos del cálculo matemático en la compresión de los fenómenos.

Para Ockham, poner en movimiento la indagación científica partiendo de principios racionalmente definidos y estructuras necesarias era perfectamente lógico en el ámbito de la física aristotélica, ya que partía del presupuesto de que todo se desarrollaba según leyes en sí inmutables que regían un mundo fruto de la necesidad; no de la libertad. Pero la novedad del cristianismo sería precisamente que el origen de todo está en la decisión absolutamente libre de su omnipotente creador, y por tanto, en la inexistencia de una legalidad metafísica universal y original, que haya de darse por sobreentendida.

Junto a la prescripción de orden empirista, Ockham invita, por tanto, a considerar hipótesis posibles, pues todo lo que es posible podría ser, como por ejemplo la existencia de otros mundos como el nuestro, de un universo infinito, de la rotación de la tierra sobre su eje,...

Como vemos, el sistema aristotélico es desechado, sin ambages, por ockham, quien, como cabía esperar, debido a su fidelidad al dato, su compromiso con lo concreto y el uso de la «navaja» negará que entre el ámbito supralunar (celeste) y el sublunar exista la diferencia substancial que Aristóteles defendía, proponiendo la concepción de un mundo homogéneo entre sus elementos estructurales. De aquí surgirá su rechazo a la animación de los cielos o a la indivisibilidad de las sustancias celestes. Y, del mismo modo, rechazará también el principio aristotélico según el cual todo lo que se mueve es movido por otro, con lo que algunos historiadores han defendido que dio el primer paso hacia el principio de inercia, que revolucionaría la física en el siglo XvII.

Finalmente, y con ello no agoto ni mucho menos el tema, es importante también señalar dos puntos que, sin duda, fueron más tarde desarrollados por los empiristas: $1^{\circ}$ el importante empeño de Ockham por proporcionar una sólida lógica de la inducción; y $2^{\circ}$ su crítica al concepto aristotélico de causa, sobre todo de causa final, que, llevado hasta sus últimas consecuencias lógicas, condujo a Nicolás de Autrecourt a hacer afirmaciones que, sólo Hume, cuatro siglos después, sería capaz de mantener.

«Respecto de las cosas sabidas por experiencia al modo como se dice que se sabe que el ruibarbo cura el cólera o que el imán atrae al hierro, sólo poseemos un hábito de hacer conjeturas, pero no certeza. Cuando se dice que tenemos certeza respecto de tales cosas en virtud de una proposición que reposa en el alma según la cual lo que ocurre en muchas ocasiones por un curso no libre es el efecto natural de ello, yo pregunto ¿Qué 
es lo que llamas una causa natural? ¿Dices por ejemplo que lo que produjo en el pasado en muchas ocasiones y produce en el presente, producirá en el futuro si permanece y es aplicado? Entonces la premisa menor no es conocida, porque admitiendo que algo fue producido en muchas ocasiones, no es, sin embargo, conocido que deba ser producido de la misma manera en el futuro ${ }^{37}$.

«Cualesquiera que sean las condiciones que suponemos puedan ser la causa de un efecto, no sabemos, evidentemente, que, cuando se pongan esas condiciones, se seguirán los efectos en cuestión $»^{38}$.

El ingenio, la audacia, el afán por descubrir y la creatividad que derrocharon los filósofos que empeñaron sus esfuerzos en repensar la formidable herencia recibida de culturas ajenas, como la griega y la musulmana, pero profundamente admiradas, merece nuestro reconocimiento. La labor llevada a cabo por estos hombres, en condiciones alejadas de nuestra comodidad, es, en sí misma, extraordinaria, y resulta asombroso que en algún momento haya podido ser minusvalorada. Y si a esto añadimos el vigoroso contenido de muchas de sus propuestas en el campo de la filosofía natural, no es extraño que los historiadores de la ciencia y del pensamiento en general que han dedicado sus esfuerzos a este período de nuestra cultura, los siglos XIII y XIV, hayan terminado, tarde o temprano, envueltos en la difícil tarea de discernir hasta qué punto lo hecho en los siglos XVI, XVII e incluso XVIII se debía a lo pensado por los hombres del final del medievo. Pero esa es otra historia y habrá de ser contada en otro lugar.

Universidad Pontificia Comillas

IgNACIO VERdú BERganZA

Instituto de Humanidades Francesco Petrarca

vonverdu@yahoo.es

[Artículo aprobado para publicación en diciembre de 2014].

37 Citado por Crombie, A. C., Historia de la ciencia..., Vol. 2, p. 38.

38 Ibidem. 